\title{
Direct vs Delayed Triplet Energy Transfer from Organic Semiconductors to Quantum Dots and Implications for Luminescent Harvesting of Triplet Excitons
}

Victor Gray, Jesse R. Allardice, Zhilong Zhang, Simon Dowland, James Xiao, Anthony J. Petty, II, John E. Anthony, Neil C. Greenham, and Akshay Rao*

Cite This: ACS Nano 2020, 14, 4224-4234

Read Online

ACCESS | Lill Metrics \& More | 国 Article Recommendations | St Supporting Information

ABSTRACT: Hybrid inorganic-organic materials such as quantum dots (QDs) coupled with organic semiconductors have a wide range of optoelectronic applications, taking advantage of the respective materials' strengths. A key area of investigation in such systems is the transfer of triplet exciton states to and from QDs, which has potential applications in the luminescent harvesting of triplet excitons generated by singlet fission, in photocatalysis and photochemical upconversion. While the transfer of energy from QDs to the triplet state of organic semiconductors has been intensely studied in recent years, the mechanism and materials parameters controlling the reverse process, triplet transfer to QDs, have not been well investigated. Here, through a combination of steadystate and time-resolved optical spectroscopy we study the mechanism and energetic dependence of triplet energy transfer from an organic ligand

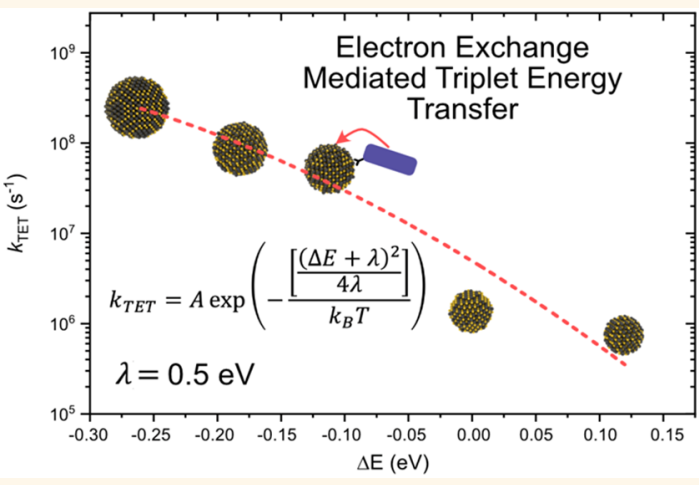
(TIPS-tetracene carboxylic acid) to PbS QDs. Over an energetic range spanning from exothermic $(-0.3 \mathrm{eV})$ to endothermic $(+0.1 \mathrm{eV})$ triplet energy transfer we find that the triplet energy transfer to the QD occurs through a single step process with a clear energy dependence that is consistent with an electron exchange mechanism as described by Marcus-Hush theory. In contrast, the reverse process, energy transfer from the QD to the triplet state of the ligand, does not show any energy dependence in the studied energy range; interestingly, a delayed formation of the triplet state occurs relative to the quantum dots' decay. Based on the energetic dependence of triplet energy transfer we also suggest design criteria for future materials systems where triplet excitons from organic semiconductors are harvested via QDs, for instance in light emitting structures or the harvesting of triplet excitons generated via singlet fission.

KEYWORDS: triplet energy transfer, singlet fission, quantum dots, photon multiplication, solar energy

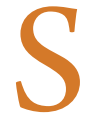

emiconductor quantum dots (QDs) are a class of materials with a wide range of possible applications. With their superb optical properties, QDs are particularly well suited as absorbing and emitting species in optoelectronic devices. ${ }^{1-8}$ Since the bright-dark state splitting in QDs is small, much smaller than $k T$ at room temperature, efficient emission with no losses due to dark spin states is common. ${ }^{9,10}$ In contrast, spin-1 triplet states in organic semiconductors are spin forbidden to relax to the ground state by photon emission; i.e., they are dark states. However, it has been shown that it is possible to transfer such dark triplet excitons from organic semiconductors to QDs, where the excitation energy subsequently can be emitted as a photon, ${ }^{11-15}$ thus converting the dark states into bright states.
Following the first demonstrations that it was possible to transfer energy from the triplet state of an organic semiconductor to a QD, ${ }^{11,12}$ there has been a growing interest in such hybrid organic-inorganic systems and their potential applications in photocatalysis and photochemical upconversion. ${ }^{16-18}$ The key photophysical process in such systems, the transfer of energy from the QD to organic, has gained

Received: November 26, 2019

Accepted: March 17, 2020

Published: March 17, 2020 

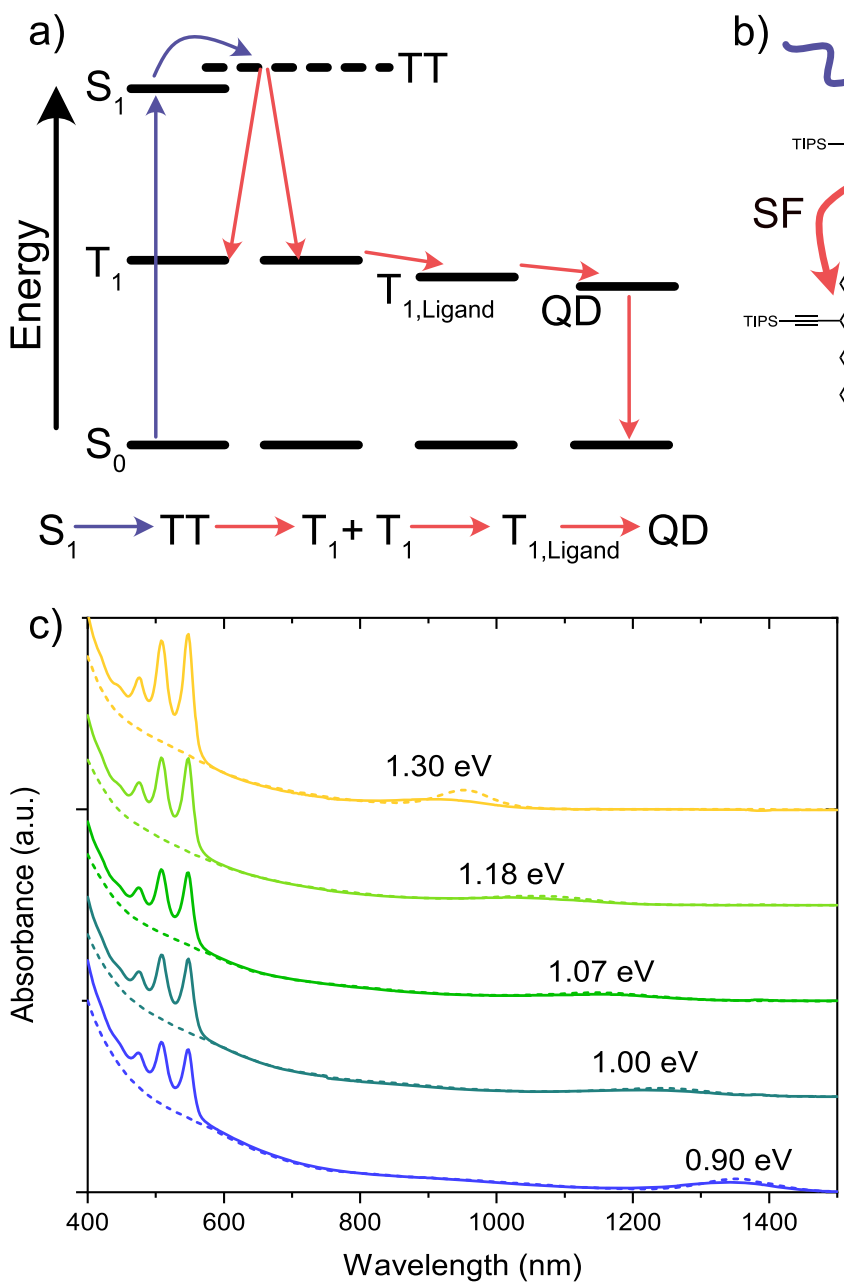
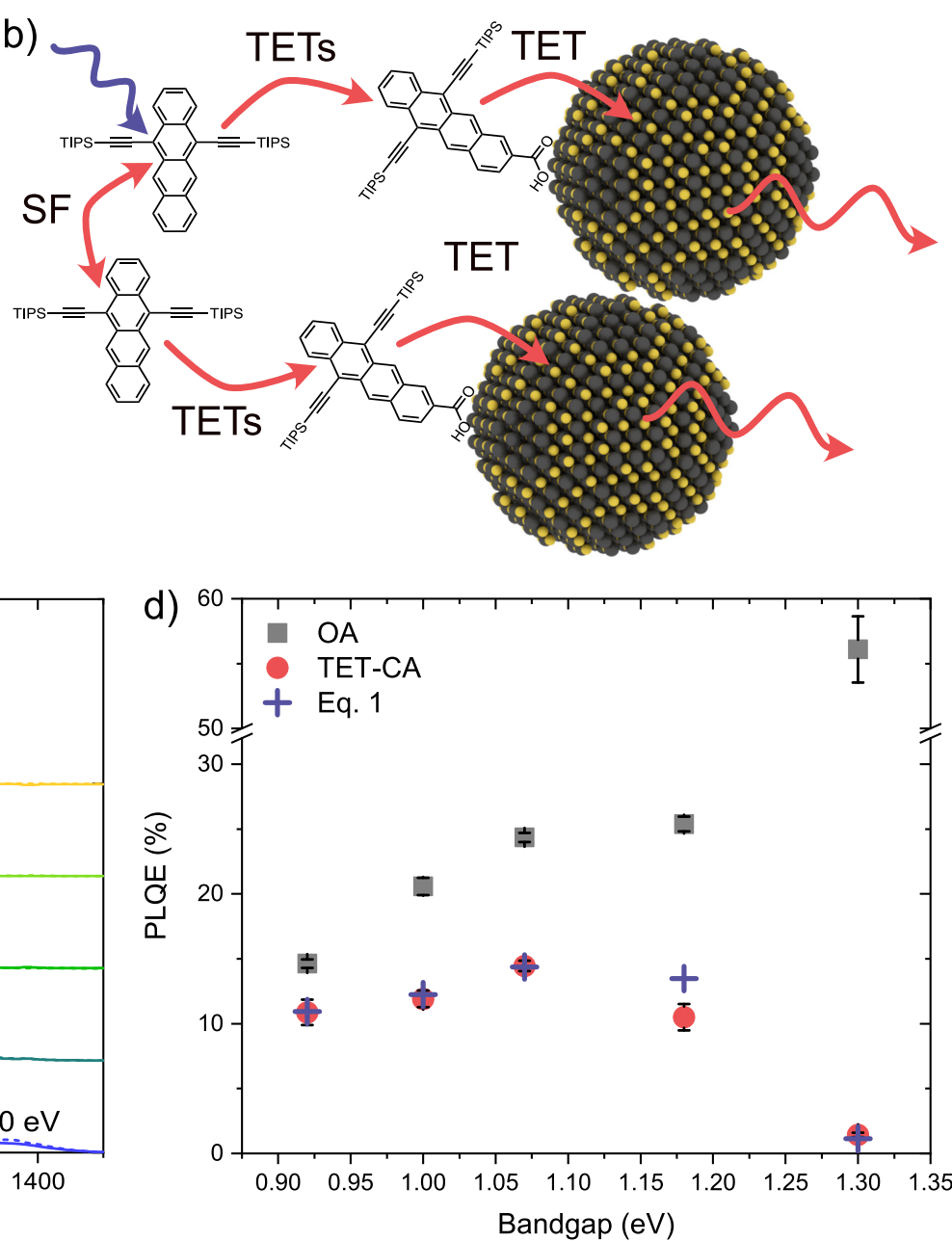

Figure 1. (a) Schematic energy diagram illustrating the singlet-fission process and photon multiplication pathway where a singlet excited state $\left(S_{1}\right)$ generates two triplet states $\left(T_{1}\right)$ via an intermediate TT state, followed by triplet energy transfer (TET) to a quantum dot (QD) via an intermediate ligand state. (b) Schematic illustration of the photon-multiplication process described in part a. (c) Absorption spectra of the series of $\mathrm{PbS}$ QDs studied herein, with excitonic band gaps ranging from 0.9 to $1.3 \mathrm{eV}$. Dotted lines are absorption from the assynthesized OA-capped PbS QDs, and solid lines are the absorption after ligand exchange with TIPS-tetracene carboxylic acid, TET-CA. (d) Photoluminescence quantum efficiencies, PLQEs, at $658 \mathrm{~nm}$ excitation of the as-synthesized OA-capped QDs (gray squares), QDs with TET-CA ligand (red circles), and calculated quantum yields from eq 1 (blue crosses).

considerable attention. ${ }^{13,16-19}$ For example, the transfer from CdSe to anthracene ligands has been studied for photon upconversion applications using both steady-state and timeresolved approaches. ${ }^{16,17,20,21}$ It is generally accepted that the triplet energy transfer in these $\mathrm{CdSe}$-anthracene materials occurs through a Dexter exchange mechanism. ${ }^{19,21,22}$ It would be expected that triplet transfer from other QDs, such as PbS and $\mathrm{PbSe}$, would occur through a similar mechanism. In some instances there is evidence supporting a similar direct transfer mechanism in $\mathrm{PbS}$-tetracene systems. ${ }^{23-25}$ Surprisingly, recent observations indicate that in other cases the triplet exciton transfer from $\mathrm{PbS}$ QDs to organic ligands occurs via an intermediate state. ${ }^{26,27}$ Luo et al. recently showed that triplet transfer from perovskite QDs to organic ligands can occur through a simultaneous or stepwise two electron process depending on the band alignment. ${ }^{28}$

In contrast, the transfer of triplet excitons to QDs has been much less studied following the initial demonstrations of these hybrid systems. The mechanism of triplet energy transfer from organics to $\mathrm{PbS}$ has not been elucidated, and the question still remains if it occurs via a direct exchange mechanism or via an intermediate state. Furthermore, in both cases (triplet transfer to and from QDs), thermodynamic considerations, with regards to the energetic driving force for triplet energy transfer, have only been reported in a few cases. ${ }^{13,25}$ Understanding the energetic requirements for exciton transfer is crucial for modeling device behavior, which in turn can directly aid in the design of future, more suitable materials. In the case of triplet transfer to QDs, this process could form the underlying working principal of LEDs based on triplet harvesting from organics and also a recently proposed device concept-the singlet fission photon multiplier (PM) - which this work will examine in some detail.

Singlet fission (SF) is a spin-allowed process in organic semiconductors forming two triplet excitons from an initial photoexcited singlet excited state (Figure 1a). ${ }^{29,30}$ As an exciton multiplication process, SF has been suggested as a possible way of overcoming thermalization losses in photovoltaic (PV) devices, increasing the theoretical maximum efficiency of a single-junction device from $33 \%$ to $44 \% .{ }^{30-32}$ A few SF-based PV devices have been demonstrated; ; $^{31,33-37}$ however, overall efficiencies are still low. Moreover, as 
suggested by Dexter 50 years ago, ${ }^{38}$ it would be a great gain if traditional Si-based PVs could be coupled with an SF material, and efforts toward this end are being made. ${ }^{39,40} \mathrm{~A}$ major difficulty is how to transfer the generated triplet excitons to the $\mathrm{Si}-\mathrm{PV}$ efficiently as the diffusion length of triplets is limited, and the efficiency of $\mathrm{Si}-\mathrm{PV}$ is sensitive to surface passivation. ${ }^{39,40}$ Emissive states, such as singlet spin-0 states, are easier to transfer long distances, either through FRET or photon emission with subsequent photon absorption in the acceptor. It has therefore been proposed that combining emissive QDs with an exciton multiplication material (such as an SF material) could constitute a photon multiplication (PM) material. ${ }^{14,15,30,32}$ The working principle of such a PM material is as follows (see Figure 1a,b): Absorption of a high-energy photon by the SF material generates two triplet excitons. These triplets are then transferred into emissive QDs, generating two excited QDs that efficiently emit two photons. The emitted photons are then absorbed in a conventional PV cell, such as Si. Examples of PM materials have been demonstrated in solution recently. ${ }^{14,15}$ Important in developing these materials further and of fundamental interest for many other optoelectronic materials is to gain a detailed understanding of the mechanism of triplet energy transfer from organic molecules to inorganic QDs.

Here, we elucidate the mechanism and energy dependence of triplet exciton transfer to QDs from organic ligands. Our model system consists of 6,11-bis((triisopropylsilyl)ethynyltetracene-2-carboxylic acid (TET-CA) ligands attached to $\mathrm{PbS} \mathrm{QDs}$, as well as 5,12-bis((triisopropylsilyl)ethynyltetracene (TIPS-Tc) in concentrated solution as a singlet-fission material to generate free triplets. The triplet transfer is studied both indirectly through steady-state absolute photoluminescence efficiency (PLQE) measurements as well as directly through transient absorption spectroscopy. We vary the QD size and hence the exciton energy to achieve systems with different driving energies for triplet transfer to the QDs, ranging from exothermic $(-0.3 \mathrm{eV})$ to endothermic $(+0.1 \mathrm{eV})$. We find that triplet energy transfer to the QD occurs via a single step process, consistent with an electron exchange mechanism with a Marcus-Hush energy dependence, in line with previous indications in solution. ${ }^{25}$ An energy offset of about $-0.1 \mathrm{eV}$ between the QD and triplet mediator ligand is required for efficient net transfer. We can accurately model the PM behavior and use the developed model to propose future design criteria for efficient PM systems suitable for Si-PVs. We conclude that SF materials with higher triplet energies (1.4$1.5 \mathrm{eV}$ ) will be required for practical applications.

\section{RESULTS/DISCUSSION}

Ligand Exchange of PbS QDs. Oleic acid (OA)-capped $\mathrm{PbS}$ quantum dots with excitonic absorption peaks from 0.9 to $1.3 \mathrm{eV}$ (Figure 1c) were synthesized according to previously reported methods. ${ }^{41}$ To achieve efficient triplet energy transfer to and from semiconductor quantum dots, a mediator ligand is often required. ${ }^{15-17}$ We therefore partly exchanged the native oleic acid ligands to TIPS-tetracene-carboxylic acid (TET-CA, Figure S1) as described previously ${ }^{15}$ (details of the ligand exchange can be found in the SI). The ligand coverage was within the range $1.6-1.9 \mathrm{TET}-\mathrm{CA} / \mathrm{nm}^{2}$ for all sizes of QDs (as determined from UV/vis absorption, Supporting Information, Figure S2 and Table S1). The absolute photoluminescence quantum yield (PLQE) of the OA-capped dots increases from $10 \%$ to almost $60 \%$ when the band gap increases from 0.9 to
$1.3 \mathrm{eV}$, shown in Figure 1d. The trend of increasing PLQE with band gap is well-known for QDs. ${ }^{42}$ After ligand exchange, the PLQE is quenched, and two distinct quenching behaviors are observed. For low-band-gap dots $(0.9-1.1 \mathrm{eV})$, the PLQE quenching is about $25-40 \%$. For band gaps above $1.1 \mathrm{eV}$, however, the quenching is even more severe, approaching $99 \%$ for $1.3 \mathrm{eV}$ dots.

From ps and ns transient absorption measurements of the QDs capped with either OA or TET-CA (Figures S3 and S4), we conclude that the quenching mechanism is 2-fold; first the ligand exchange introduces some surface traps which are rapidly populated within hundreds of picoseconds after QD excitation. These traps can repopulate the $\mathrm{QD}$ excitonic state and only result in a net quenching of roughly $30 \%$. Second, in the case when the QD band gap is close to or larger than the $T_{1}$ energy of the TET-CA ligand (approximately $1.2 \mathrm{eV}$, see below), triplet energy transfer (TET) from the $\mathrm{PbS} \mathrm{QD}$ to TET-CA is possible, resulting in more significant quenching. Based on the dual quenching mechanism, we can derive an expression for the QD PLQE, eq 1 (see the SI for the derivation):

$$
\Phi_{\mathrm{QD}}=\frac{k_{\mathrm{r}}}{k_{\mathrm{r}}+k_{\mathrm{nr}}+k_{-\mathrm{TET}}+k_{\mathrm{q}}-k_{\mathrm{TET}}\left(\frac{k_{-\mathrm{TET}}}{k_{\mathrm{TET}}+k_{\mathrm{LD}}}\right)-k_{-\mathrm{q}}\left(\frac{k_{\mathrm{q}}}{k_{\mathrm{Tr}}+k_{-\mathrm{q}}}\right)}
$$

where $k_{\mathrm{r}}$ and $k_{\mathrm{nr}}$ are the radiative and nonradiative decay constants of the QDs, respectively. $k_{\mathrm{TET}}$ and $k_{-\mathrm{TET}}$ are the rates of triplet energy transfer to and from the QD, respectively. Similarly, $k_{\mathrm{q}}$ and $k_{-\mathrm{q}}$ are the rates of quenching to the trap state and $\mathrm{QD}$ repopulation rate from the trap, respectively. $k_{\mathrm{LD}}$ and $k_{\mathrm{Tr}}$ are the decay constants for the ligand triplet and QD trap state, respectively. The rate constants are also defined in the Jablonski diagram in Figure 4, which describes the studied PM system in whole. For the three low-energy QDs the triplet transfer rates $\left(k_{\text {TET }}\right.$ and $\left.k_{-\mathrm{TET}}\right)$ can be neglected in the case where the QDs are directly excited, as the uphill transfer to the ligand is slow compared to the quenching. Therefore, the quenching of the ps TA dynamics seen in Figure $\mathrm{S} 3 \mathrm{a}-\mathrm{c}$ solely originates from trap quenching. From these decays we approximate the trap quenching rate to $k_{\mathrm{q}} \approx 1.0 \times 10^{10} \mathrm{~s}^{-1}$. We find that $k_{-\mathrm{q}}=0.3 \times 10^{10} \mathrm{~s}^{-1}$ and $k_{\mathrm{Tr}}=1.0 \times 10^{5} \mathrm{~s}^{-1}$ reproduce the quenched PLQE values in Figure $1 \mathrm{~d}$ for QDs with band gaps 0.9-1.07 eV. For the higher-energy QDs (band gaps $1.18-1.3 \mathrm{eV}$ ), there is the additional quenching via triplet energy transfer, and the quenching seen in the decays in Figure S3 cannot exclude contributions from TET. However, by assuming that the trap quenching is constant over the band gap range studied here and with triplet energy transfer rates $\left(k_{\text {TET }}\right.$ and $k_{-\mathrm{TET}}$ ) determined from our ns TA measurements (see below), we can accurately reproduce the PLQEs of all the ligand-exchanged QDs (Figure 1d). The excellent agreement to the experimental PLQEs allows us for further modeling to assume that the trap quenching and repopulation dynamics do not change significantly with band gap, but only an additional quenching by triplet transfer to the ligand appears when it becomes energetically favorable.

Singlet-Fission and Triplet Energy Transfer to PbS QDs in Solution. We choose TIPS-tetracene (TIPS-Tc, Figure S1) as the SF material as it is an extremely soluble tetracene derivative that is known to undergo SF in both concentrated solution and films. ${ }^{15,43,44}$ In solution, the free triplet yield has been reported to be $120-140 \%{ }^{15,43}$ at 
concentrations of $200 \mathrm{mg} / \mathrm{mL}(0.34 \mathrm{M})$. Figure 2a displays the PLQE of the TET-CA covered dots in a $200 \mathrm{mg} / \mathrm{mL}$ TIPS-Tc
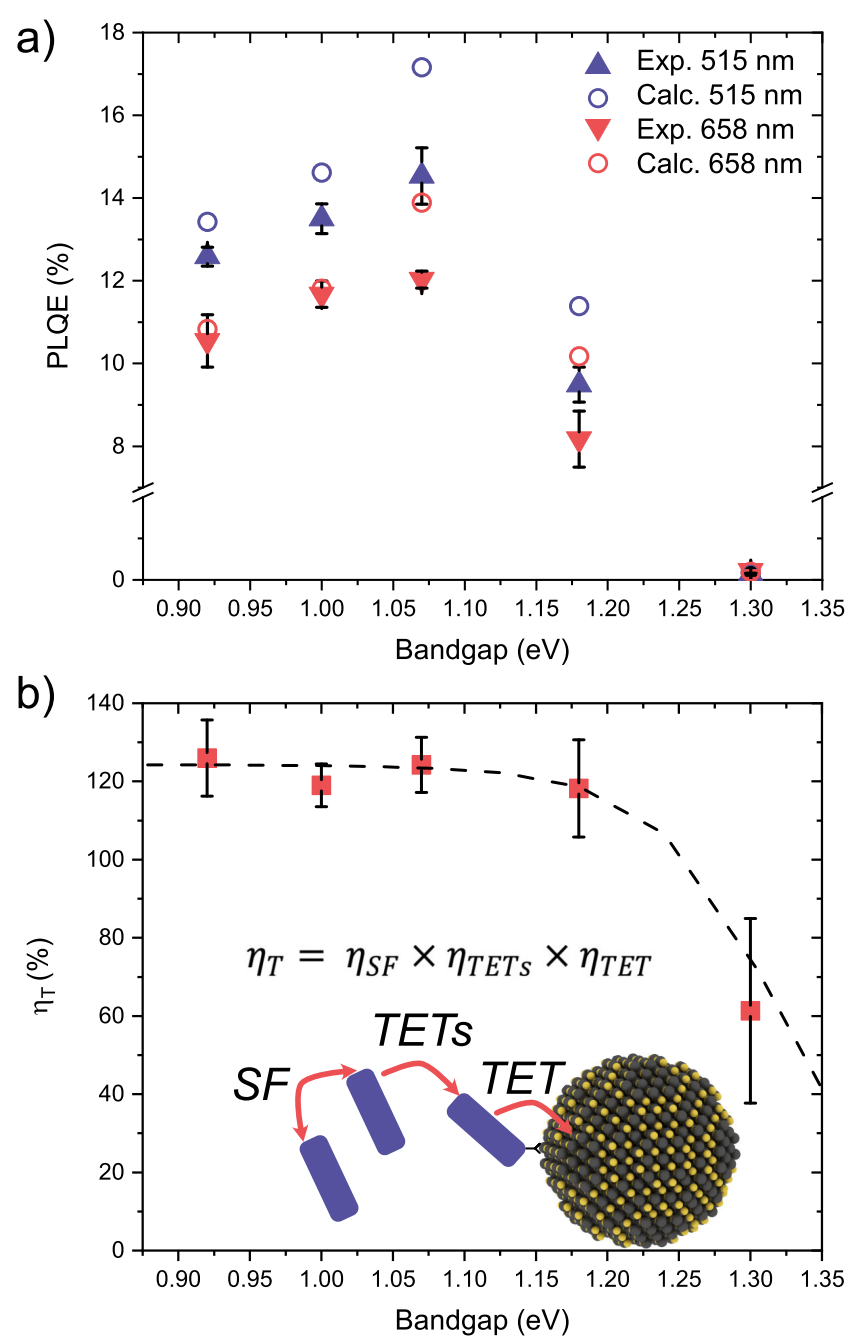

Figure 2. (a) Measured and modeled photoluminescence quantum efficiencies (PLQEs) of PbS quantum dots (QDs) of various band gaps. Samples are $0.2 \mathrm{mM}$ QDs with TET-CA ligands in $200 \mathrm{mg}$ / $\mathrm{mL}$ TIPS-Tc solutions. Excitation with $515 \mathrm{~nm}$ mainly excites the singlet-fission material TIPS-Tc, and excitation at $658 \mathrm{~nm}$ directly excites the $\mathrm{PbS}$ QDs. (b) Triplet transfer efficiency $\left(\eta_{\mathrm{T}}\right)$ from TIPS-Tc to the QDs, determined from the PLQE enhancement following eq 2; dashed line is the theoretical triplet transfer efficiency obtained from the kinetic modeling described below. Inset shows the schematic energy transfer process and the equation highlights that the triplet transfer efficiency is a product of the singlet-fission (SF) yield, the efficiency of triplet transfer in solution (TETs), and the efficiency of triple transfer from the ligand to the QD (TET).

solution, exciting either TIPS-Tc $(515 \mathrm{~nm})$ or the QDs (658 $\mathrm{nm})$. For QDs with energies $<1.25 \mathrm{eV}$ there is an enhancement of the PLQE when the singlet-fission material TIPS-Tc is excited, suggesting exciton multiplication through SF followed by triplet energy transfer (TET) to the QDs. From the PLQE values in Figure $2 \mathrm{a}$ and taking into account the fractional absorption of the two components in the samples, we estimate the number of triplets transferred to a QD per photon absorbed $\left(\eta_{\mathrm{T}}\right)$ according to eq $2:^{12,15}$

$$
\eta_{\mathrm{T}}=\left(\frac{\Phi_{\mathrm{PM}}}{\Phi_{\mathrm{QD}}}-\frac{\mathrm{Abs}_{\mathrm{QD}}^{515 \mathrm{~nm}}}{\mathrm{Abs}_{\mathrm{Tot}}^{515 \mathrm{~nm}}}\right)\left(\frac{\mathrm{Abs}_{\mathrm{TIPS}-\mathrm{Tc}}^{515 \mathrm{~nm}}}{\mathrm{Abs}_{\mathrm{Tot}}^{515 \mathrm{~nm}}}\right)^{-1}
$$

where $\Phi_{\mathrm{PM}}$ is the measured PLQE when exciting the SF material TIPS-Tc, and $\Phi_{\mathrm{QD}}$ is the PLQE when exciting the QD directly. The total absorption at the excitation wavelength $515 \mathrm{~nm}\left(\mathrm{Abs}_{\mathrm{Tot}}^{515 \mathrm{~nm}}\right)$ is the sum of the absorption of the two components TIPS-Tc $\left(\mathrm{Abs}_{\mathrm{TIPS}-\mathrm{Tc}}^{515 \mathrm{~nm}}\right)$ and $\mathrm{QD}\left(\mathrm{Abs}_{\mathrm{QD}}^{515 \mathrm{~nm}}\right)$ estimated from the concentrations and molar absorption coefficients. In Figure $2 \mathrm{~b}$ it can be seen that the triplet transfer efficiency is $120 \pm 5 \%$ for QDs with energies $<1.25 \mathrm{eV}$. Our observations are in line with a triplet yield of $130 \pm 10 \%$ in TIPS-Tc solutions followed by close to quantitative TET to the QDs, as we have recently reported. ${ }^{15}$

To further study the triplet energy transfer dynamics, we turn to ns-resolved transient absorption (TA) spectroscopy. Transient absorption spectra of TIPS-Tc solutions excited at $515 \mathrm{~nm}$ correspond well to previous reports, with the characteristic triplet absorption peaks at 860 and $970 \mathrm{~nm}$ (Figure 3a). ${ }^{15,43}$ Analyzing the kinetics of SF in our TIPS-Tc solutions, we conclude that the triplet decay occurs mainly though intrinsic first-order decay at fluences below $4 \mu \mathrm{J} / \mathrm{cm}^{2}$, with a rate constant, $k_{\mathrm{Tc}}$, of $1.1 \times 10^{4} \mathrm{~s}^{-1}$ (Figure S5). When TET-CA covered PbS QDs are added to the TIPS-Tc solution, a quenching of the triplet signal is observed, as shown Figure $3 \mathrm{~b}$. The TIPS-Tc is quenched by up to $95 \%$ by the $0.9 \mathrm{eV}$ QD/TET-CA system, suggesting efficient TET from TIPS-Tc to the QDs and supporting the steady-state PLQE measurements. Another telling sign of TET to the QDs is the fact that the QD lifetimes are extended when TIPS-Tc is excited, compared to directly exciting the QDs at $658 \mathrm{~nm}$ (Figure 3c,d and Figure S6). The extended lifetime arises due to the delayed formation of QD excitons from TET.

We decompose the QD features and the TIPS-Tc triplet features from the combined TA maps, as described in the SI. In this way the kinetics of the QD and TIPS-Tc triplet populations are obtained. To recover the QD population originating from TET from TIPS-Tc, the intrinsic QD kinetics, obtained by direct excitation at $658 \mathrm{~nm}$, are subtracted from the decomposed QD kinetics at $515 \mathrm{~nm}$ excitation. Figure $4 \mathrm{~b}$ and Figure S7 show these extracted kinetic traces where a delayed growth of the QD ground state bleach is clearly observed in the time scale of TET for QDs with exciton energies below $1.25 \mathrm{eV}$. For the $1.3 \mathrm{eV}$ QD there is no QD ground state bleach in the time-resolution of our experiment. In fact, when the $1.3 \mathrm{eV} \mathrm{QD}$ is excited directly, there is a growth of the ligand triplet feature until $1 \mu \mathrm{s}$ (Figure S8). For the $1.3 \mathrm{eV} \mathrm{QD}$, there is no $\mathrm{QD}$ ground state bleach in the timeresolution of our experiment. In fact, when the $1.3 \mathrm{eV} \mathrm{QD}$ is excited directly, there is a growth of the ligand triplet feature until $1 \mu$ s (Figure S8). Considering that QD ground state bleach of the $1.3 \mathrm{eV}$ QD/TET-CA recovers up to $95 \%$ in the first $2 \mathrm{~ns}$, the delayed triplet formation could indicate a stepwise TET process or a competition of two separate processes: trap quenching and triplet transfer. In $\mathrm{PbS}-$ pentacene systems, reports suggest either a charge-separated or a localized surface state intermediate giving rise to a delayed triplet formation; ${ }^{26,27}$ however, a stepwise TET process has not been observed in previous $\mathrm{PbS}$-tetraene systems in the literature. ${ }^{23-25}$ The reason for the delayed triplet formation is currently unknown. We did not observe any electroabsorption features, which have been associated with surface states, or 

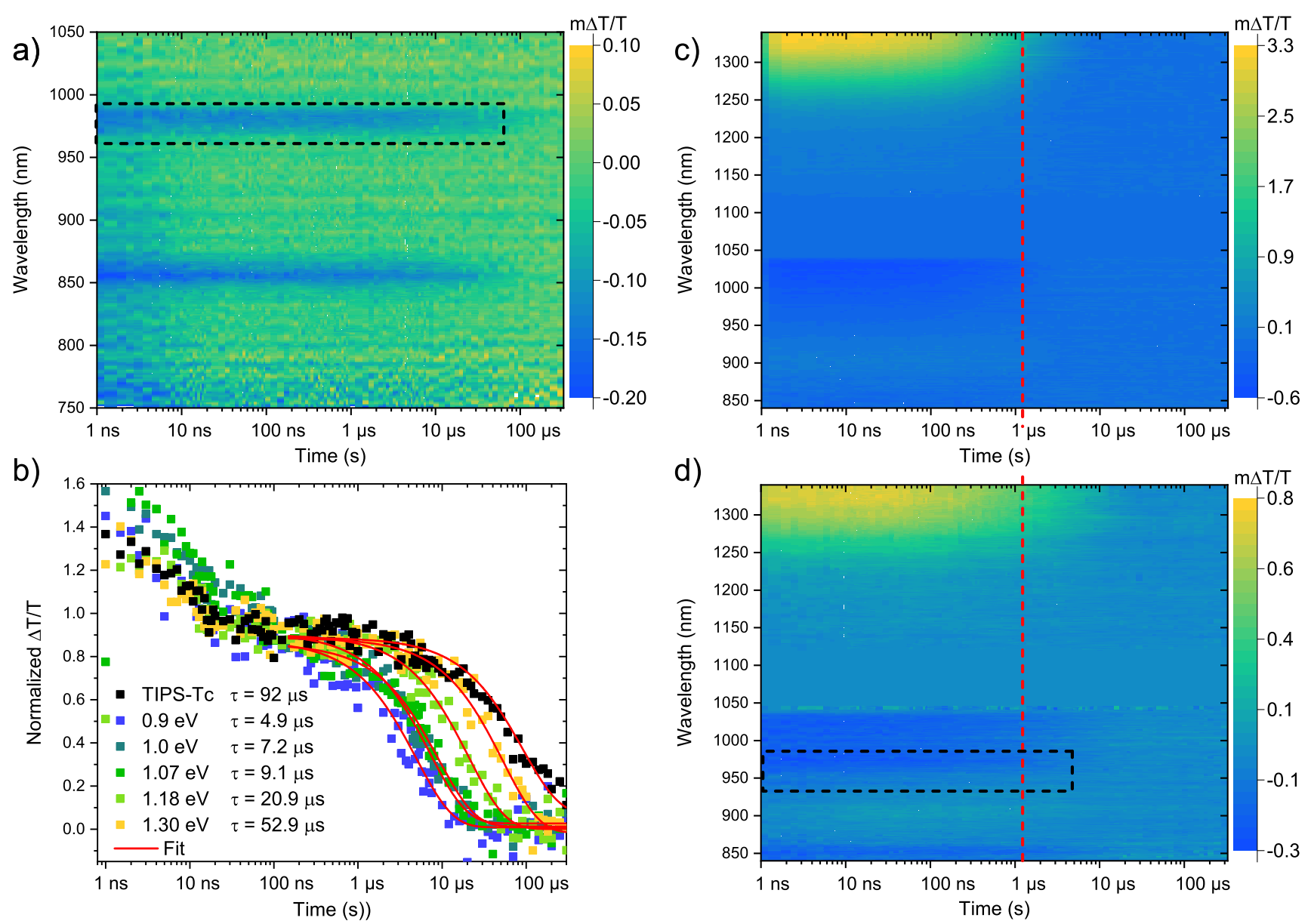

Figure 3. (a) Transient absorption (TA) map of a concentrated TIPS-Tc solution $(200 \mathrm{mg} / \mathrm{mL})$ excited at $515 \mathrm{~nm}\left(4 \mu \mathrm{J} / \mathrm{cm}^{2}\right)$ with clear triplet absorption features at 855 and $970 \mathrm{~nm}$. (b) Triplet decay kinetics of the TIPS-Tc solutions in part a compared with TIPS-Tc solutions containing PbS quantum dots $(2 \mathrm{mM})$ covered with TET-CA ligands. Legend shows fitted monoexponential lifetimes. (c) TA map of $0.9 \mathrm{eV}$ QDs with TET-CA ligands in $200 \mathrm{mg} / \mathrm{mL}$ TIPS-Tc, excited directly at $650 \mathrm{~nm}, 3 \mu \mathrm{J} / \mathrm{cm}^{2}$. (d) TA map of $0.9 \mathrm{eV}$ QDs in part c but exciting TIPS-Tc at $515 \mathrm{~nm}\left(4 \mu \mathrm{J} / \mathrm{cm}^{2}\right)$. Dashed box highlights triplet signal at $970 \mathrm{~nm}$. Red dashed line is a guide for the eye highlighting the extension in QD lifetime in part $\mathrm{d}$ due to triplet transfer from TIPS-Tc.

signals associated with a charged intermediate in our TA data. Future studies with other techniques, such as electron spin resonance, may provide answers by examining the spin states involved in the process and identifying possible intermediates. Further, in the presence of TIPS-Tc, both the 1.3 and $1.18 \mathrm{eV}$ QDs show evidence of triplet transfer from the QD, via the ligand, to TIPS-Tc (Figure S6). Since the $1.18 \mathrm{eV}$ QD can both accept and donate triplets from/to the ligand, we conclude that the TET-CA $T_{1}$ energy is close to resonant to the $1.18 \mathrm{eV}$ QD. Thus, TET-CA has a slightly lower $T_{1}$ energy than TIPS-Tc, by approximately $0.1 \mathrm{eV}$, allowing for an efficient energy cascade into lower-energy dots.

Even though the isoenergetic system of $1.18 \mathrm{eV}$ QDs with TET-CA ligands has a high triplet transfer efficiency of $120 \%$ (Figure 2b), the absolute PLQE of the $1.18 \mathrm{eV}$ QD/TET-CA system is lower than the lower-energy QDs. The possibility for exciton equilibration between $\mathrm{QD}$ and ligand states in the isoenergetic system would explain this lower PLQE. Thus, we can conclude that even though efficient triplet transfer can occur in the isoenergetic system, the QD/TET-CA exciton equilibrium leads to the need for an energy offset of $0.1 \mathrm{eV}$ to achieve an overall efficient PM system. By modeling the triplet transfer dynamics in the PM systems we hope to gain a better understanding of the triplet transfer dependence on energetic driving force $(\Delta E)$ to aid future design of hybrid organicinorganic optoelectronic materials.

Kinetic Model of PM and Fitting of Steady-State and Transient Data. We can now build a model describing the PM system. The Jablonski diagram in Figure 4 illustrates the states and processes involved, including possible quenching routes. In the following description, the rate constants of the described process are given in brackets. The first step that needs to happen is the absorption of a photon $\left(k_{\mathrm{Ex}} / \mathrm{s}^{-1}\right)$ by TIPS-Tc to form the first excited singlet state ${ }^{1}$ TIPS-Tc*. From here, singlet fission $\left(k_{\mathrm{TT}} / \mathrm{s}^{-1}\right)$ forming a correlated triplet pair (TT) state, involving two TIPS-Tc molecules, competes with intrinsic radiative and nonradiative singlet decay $\left(k_{\mathrm{SD}} /\right.$ $\mathrm{s}^{-1}$ ). At high concentrations the formation of TT can be considered to be pseudo-first-order in [ ${ }^{1}$ TIPS-Tc* ${ }^{*}$. The TT state can dissociate $\left(k_{\mathrm{SF}} / \mathrm{s}^{-1}\right)$ into two free triplets, ${ }^{3}$ TIPS-Tc*. However, the TT state can also decay through radiative and nonradiative pathways $\left(k_{\mathrm{TTD}} / \mathrm{s}^{-1}\right)$. Triplet transfer from ${ }^{3}$ TIPS-Tc* to the ligand $\left(k_{\mathrm{sTET}} / \mathrm{M}^{-1} \mathrm{~s}^{-1}\right)$ generates the triplet excited ligand ${ }^{3}$ TET-CA*. The triplet exciton can then either be transferred to the QD $\left(k_{\mathrm{TET}} / \mathrm{s}^{-1}\right)$ or back to a TIPS-Tc molecule $\left(k_{-\mathrm{sTET}} / \mathrm{M}^{-1} \mathrm{~s}^{-1}\right)$. Both ${ }^{3}$ TIPS-Tc* and ${ }^{3}$ TET-CA ${ }^{*}$ can decay through intrinsic triplet decay channels, $\left(k_{\mathrm{TD}} / \mathrm{s}^{-1}\right)$ and $\left(k_{\mathrm{LD}} / \mathrm{s}^{-1}\right)$, respectively. As discussed above, there are a 

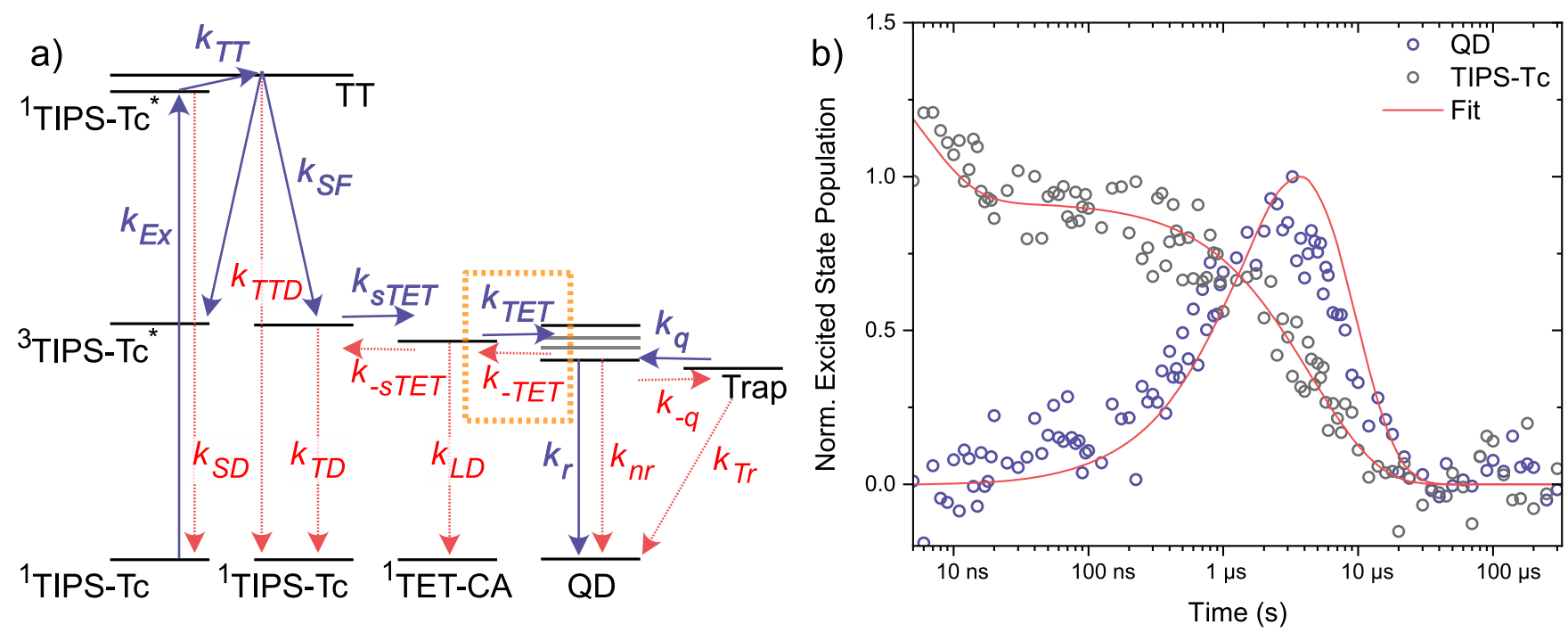

Figure 4. (a) Jablonski diagram illustrating the excited states involved in the photon multiplication (PM) process. Processes leading toward the desired quantum dot (QD) excited state are colored in blue; detrimental relaxation and quenching processes are shown as red dotted lines. Rate constants for a process are shown next to the corresponding arrow. The unknown rate constants $k_{\mathrm{TET}}$ and $k_{-\mathrm{TET}}$ determined from the fitting are highlighted in the orange dashed box. (b) Excited state population kinetics of TIPS-Tc triplets (black circles) and 0.9 eV quantum dots (QDs, blue circles) obtained by decomposing the QD and TIPS-Tc only spectra from the transient absorption maps in Figure 3. Red line shows the fitted population dynamics.

Table 1. Rate Constants Used for Fitting the Kinetic Model in Equations S5-S13; Rate Constants Are Defined in Figure 4

\begin{tabular}{|c|c|c|c|c|c|}
\hline & $0.9 \mathrm{eV}$ & $1.0 \mathrm{eV}$ & $1.07 \mathrm{eV}$ & $1.18 \mathrm{eV}$ & $1.30 \mathrm{eV}$ \\
\hline$k_{\mathrm{Ex}}^{a}\left(\mathrm{~s}^{-1}\right)$ & $1.8 \times 10^{-4}$ & $1.8 \times 10^{-4}$ & $1.8 \times 10^{-4}$ & $1.8 \times 10^{-4}$ & $1.8 \times 10^{-4}$ \\
\hline$k_{\mathrm{Ex}-\mathrm{SS}}^{b}\left(\mathrm{~s}^{-1}\right)$ & 0.031 & 0.032 & 0.034 & 0.035 & 0.037 \\
\hline$k_{\mathrm{ExQD}-515 \mathrm{~nm}}^{c}\left(\mathrm{~s}^{-1}\right)$ & 1.01 & 0.91 & 0.67 & 0.62 & 0.29 \\
\hline$k_{\text {ExQD-658nm }} d\left(\mathrm{~s}^{-1}\right)$ & 20.2 & 20.2 & 20.2 & 20.2 & 20.2 \\
\hline$k_{\mathrm{SD}}^{e}\left(\mathrm{~s}^{-1}\right)$ & $6.8 \times 10^{7}$ & $6.8 \times 10^{7}$ & $6.8 \times 10^{7}$ & $6.8 \times 10^{7}$ & $6.8 \times 10^{7}$ \\
\hline$k_{\mathrm{TT}}^{f}\left(\mathrm{~s}^{-1}\right)$ & $5.6 \times 10^{9}$ & $5.6 \times 10^{9}$ & $5.6 \times 10^{9}$ & $5.6 \times 10^{9}$ & $5.6 \times 10^{9}$ \\
\hline$k_{\mathrm{SF}}^{g}\left(\mathrm{~s}^{-1}\right)$ & $1.11 \times 10^{8}$ & $1.11 \times 10^{8}$ & $1.11 \times 10^{8}$ & $1.11 \times 10^{8}$ & $1.11 \times 10^{8}$ \\
\hline$k_{\mathrm{TTD}}^{e}\left(\mathrm{~s}^{-1}\right)$ & $5.74 \times 10^{7}$ & $5.74 \times 107$ & $5.74 \times 10^{7}$ & $5.74 \times 10^{7}$ & $5.74 \times 10^{7}$ \\
\hline$k_{\mathrm{TD}}\left(\mathrm{s}^{-1}\right)$ & $1.1 \times 10^{4}$ & $1.1 \times 10^{4}$ & $1.1 \times 10^{4}$ & $1.1 \times 10^{4}$ & $1.1 \times 10^{4}$ \\
\hline$k_{\mathrm{sTET}}\left(\mathrm{s}^{-1} \mathrm{M}^{-1}\right)$ & $1 \times 10^{9}$ & $1 \times 10^{9}$ & $1 \times 10^{9}$ & $1 \times 10^{9}$ & $1 \times 10^{9}$ \\
\hline$k_{-\mathrm{sTET}}\left(\mathrm{s}^{-1} \mathrm{M}^{-1}\right)$ & $1 \times 10^{7}$ & $1 \times 10^{7}$ & $1 \times 10^{7}$ & $1 \times 10^{7}$ & $1 \times 10^{7}$ \\
\hline$k_{\mathrm{LD}}\left(\mathrm{s}^{-1}\right)$ & $3.45 \times 10^{4}$ & $3.45 \times 10^{4}$ & $3.45 \times 10^{4}$ & $3.45 \times 10^{4}$ & $3.45 \times 10^{4}$ \\
\hline$k_{\mathrm{TET}}^{h}\left(\mathrm{~s}^{-1}\right)$ & $2 \times 10^{8}$ & $9 \times 10^{7}$ & $5 \times 10^{7}$ & $1.5 \times 10^{6}$ & $0.7 \times 10^{6}$ \\
\hline$k_{-\mathrm{TET}}^{h}\left(\mathrm{~s}^{-1}\right)$ & $2 \times 10^{7}$ & $2 \times 10^{7}$ & $1 \times 10^{7}$ & $0.4 \times 10^{7}$ & $4 \times 10^{8}$ \\
\hline$k_{\mathrm{QD}}=k_{\mathrm{r}}+k_{\mathrm{nr}}\left(\mathrm{s}^{-1}\right)$ & $1.0 \times 10^{6}$ & $0.50 \times 10^{6}$ & $0.50 \times 10^{6}$ & $0.50 \times 10^{6}$ & $0.42 \times 10^{6}$ \\
\hline$k_{\mathrm{q}}\left(\mathrm{s}^{-1}\right)$ & $1.0 \times 10^{10}$ & $1.0 \times 10^{10}$ & $1.0 \times 10^{10}$ & $1.0 \times 10^{10}$ & $1.0 \times 10^{10}$ \\
\hline$k_{-q}\left(s^{-1}\right)$ & $0.3 \times 10^{10}$ & $0.3 \times 10^{10}$ & $0.3 \times 10^{10}$ & $0.3 \times 10^{10}$ & $0.3 \times 10^{10}$ \\
\hline$k_{\mathrm{Tr}}\left(\mathrm{s}^{-1}\right)$ & $1.0 \times 10^{5}$ & $1.0 \times 10^{5}$ & $1.0 \times 10^{5}$ & $1.0 \times 10^{5}$ & $1.0 \times 10^{5}$ \\
\hline
\end{tabular}

${ }^{a}$ Rate of excitation for the time-dependent solution, $k_{\mathrm{Ex}}$ is used to calculate the initial concentration of TIPS-Tc singlets and then set to 0 for the numerical solution. Estimated from the experimental excitation density $4 \mu \mathrm{J} / \mathrm{cm}^{2} /$ pulse ${ }^{b}$ Rate of excitation of TIPS-Tc only used for the steadystate solution for calculating PLQEs, estimated from $800 \mu \mathrm{W} / \mathrm{cm}^{2}$ excitation density. ${ }^{c}$ The rate of direct excitation of the QD at $515 \mathrm{~nm}$, considering the competitive absorption between TIPS-Tc and QD, estimated from $800 \mu \mathrm{W} / \mathrm{cm}^{2}$ excitation density, only used for the steady-state solution to calculate the PLQEs. ${ }^{d}$ Excitation rate of QDs at $658 \mathrm{~nm}$ excitation used for the steady-state calculations, estimated from the experimental excitation density $8 \mathrm{~mW} / \mathrm{cm}^{2}$. ${ }^{e}$ Taken from Stern et al. ${ }^{39} f_{\text {Value interpolated from concentration dependent rates in Stern et al. }}{ }^{6}$ ${ }^{g}$ Value from Stern et al. ${ }^{39}$ multiplied by 1.3 , in line with a $130 \%$ SF yield. ${ }^{h}$ Free parameters used in the fitting.

number of quenching pathways in the QD that compete with radiative emission $\left(k_{\mathrm{r}} / \mathrm{s}^{-1}\right)$ : back triplet transfer to the ligand $\left(k_{-\mathrm{TET}} / \mathrm{s}^{-1}\right)$, quenching to a surface trap $k_{\mathrm{q}} / \mathrm{s}^{-1}$, and intrinsic nonradiative decay $\left(k_{\mathrm{nr}} / \mathrm{s}^{-1}\right)$.

Eight coupled differential rate equations govern the abovedescribed system. The rate equations are defined in the SI as Equations S5-S13 together with a detailed description of the fitting analysis, and only a brief account of the modeling is given here in the main text. Using rate constants from Stern et al., ${ }^{43}$ the kinetics of the ${ }^{3}$ TIPS-Tc* species, extracted from the TA measurement for a concentrated TIPS-Tc solution, can be accurately reproduced. The initial fast singlet-fission process will not be affected by the addition of QDs, ${ }^{15}$ and we therefore use the rate constants from Stern et al. ${ }^{43}$ for our modeling. However, we use a slightly larger $k_{\mathrm{SF}}$ (factor of 1.3) to obtain a singlet-fission triplet yield of $130 \%$ which is in accordance with previous work. ${ }^{15,43}$ From the ps-TA measurements of the ligand-exchanged QDs we can also estimate rates for the trap 
quenching and repopulation rates. For our modeling we use an average rate of trap quenching $k_{\mathrm{q}}$ of $1 / 100 \mathrm{ps}^{-1}$ and a $k_{\mathrm{q}} / k_{-\mathrm{q}}$ ratio of 1.7 to accurately reproduce the PLQE data in Figure 2 for all QD sizes. The remaining unknowns in our system are the triplet energy transfer rates: $k_{\mathrm{TET}}, k_{-\mathrm{TET}}, k_{\mathrm{STET}}$, and $k_{-\mathrm{STET}}$. To reduce the number of unknowns for the fitting, we set $k_{\text {sTET }}$ to a reasonable value for substituted acenes in toluene ${ }^{15,45}$ of 1 $\times 10^{9} \mathrm{M}^{-1} \mathrm{~s}^{-1}$. Using a detailed balance and microscopic reversibility of the rates, the reverse rate constant $k_{-s \text { TET }}$ is determined to be $1 \times 10^{7} \mathrm{M}^{-1} \mathrm{~s}^{-1}$ based on the energetic offset of $0.1 \mathrm{eV}$ between the ligand TET-CA and TIPS-Tc. To determine the triplet transfer rates $k_{\mathrm{TET}}$ and $k_{-\mathrm{TET}}$, we solve Equations S5-S13 numerically and fit the results to the kinetic traces in Figure 4b and Figure S7; see the SI for details. The fit is also constrained by the added criterion that the steady-state PLQE values should be reproduced within $20 \%$ relative of the experimental values. Table 1 summarizes the obtained fitting parameters, and Figure $4 \mathrm{~b}$ and Figure S7 show the resulting fits. Errors of the fitted parameters are estimated by varying the obtained rate constants 2 orders of magnitude while keeping other constants fixed and monitoring the goodness of the fit. The error is then taken as the values that maintain the goodness of fit within $\pm 5 \%$ of the minimum while also maintaining the PLQE within $20 \%$ of the experimental value.

The rate of triplet transfer from the QD to ligand did not show any clear trend with energetic driving force; $k_{- \text {TET }}$ was consistently around $10^{7} \mathrm{~s}^{-1}$ except for the highest-energy QD $(1.30 \mathrm{eV})$ which was an order of magnitude faster (Table 1 and Figure S9). The lack of a clear trend could indicate that the triplet energy transfer from the QD to ligand does not proceed directly but is rather mediated by an intermediate, ratedetermining step as observed for pentacene ligands. ${ }^{26,27}$ However, an indirect mechanism was not suggested by Kroupa et al. in the PbS-TET-CA system they studied. ${ }^{25}$ However, we note that their ps-TA data indicate a quick ground state recovery of the QDs and a slower triplet feature growth, as also observed by us (Figures S4e and S8).

Interestingly, the reverse process, namely, triplet transfer from the ligand to $\mathrm{QD}$, does not seem to be mediated by an intermediate rate-determining step. Instead, $k_{\text {TET }}$ shows a typical Marcus-Hush-like behavior with energetic driving force, as expected for an electron exchange mechanism. ${ }^{46-49}$ Such energy dependence is in contradiction to the initial report of resonant energy transfer to $\mathrm{PbSe}$ from a pentacene film. ${ }^{11}$ However, our findings are in line with the previous reports for CdSe QDs with pyrene ligands. ${ }^{13}$ The small band gap of pentacene, the relative band alignment with $\mathrm{PbSe}$, and the bilayer structure could allow for competing electron transfer processes possibly interfering with the TET process, explaining the difference in energy dependence. Figure 5 shows the extracted $k_{\text {TET }}$ values plotted as a function of energetic offset $\left(\Delta E\right.$ ) between the TET-CA ligand $T_{1}$ energy (taken to be 1.18 $\mathrm{eV}$ ) and the QD exciton peak. The relatively slow rates for TET indicate that the coupling between the ligand and QD is weak, suggesting that Marcus-Hush theory is a reasonable description of the energetic dependence for TET. ${ }^{50}$ The error in $k_{\mathrm{TET}}$ increases for the more exothermic systems; we suspect the reason is that the triplet transfer to the dot is no longer the rate-limiting step and becomes difficult to estimate solely from kinetic modeling. The dashed line in Figure 5 is a fit to eq 3 according to Marcus-Hush theory for electron and energy transfer: ${ }^{46-49,51,52}$

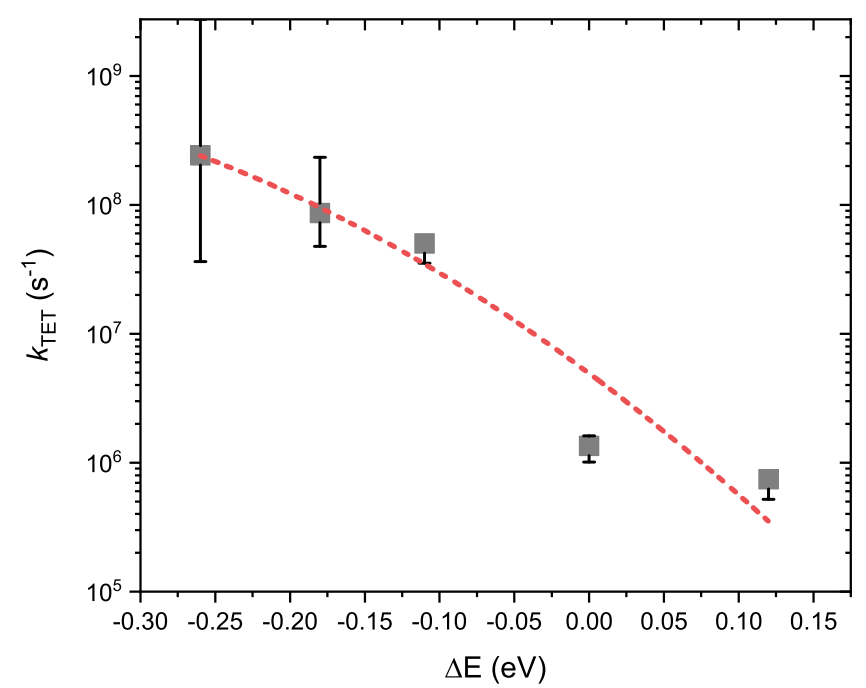

Figure 5. Obtained rate constants $k_{\mathrm{TET}}$ from the fitting to transient absorption and photoluminescence measurements, plotted as a function of energetic offset $(\Delta E)$ between ligand TET-CA and QD band gap. Dashed red line is a fit to Marcus-Hush theory for electron transfer reactions (eq 3 ).

$$
k_{\mathrm{TET}}=A \exp \left(-\frac{\left[\frac{(\Delta E+\lambda)^{2}}{4 \lambda}\right]}{k_{\mathrm{B}} T}\right)
$$

Here, $A$ is a pre-exponential factor related to the probability of transfer which can be expressed in terms of the coupling (I $\left.\left.V\right|^{2}\right)$ between the initial and final state as $A=\frac{2 \pi|V|^{2}}{\hbar \sqrt{4 \pi \lambda k_{\mathrm{B}} T}} \cdot \lambda$ is the reorganization energy, $k_{\mathrm{B}}$ the Boltzmann constant, and $T$ the temperature. The fit to eq 3 returns a reorganization energy $\lambda$ of $0.53 \mathrm{eV}$ and a pre-exponential factor of $1 \times 10^{9} \mathrm{~s}^{-1}$, similar to values reported for TET in semiconducting polymers. ${ }^{53,54}$ By obtaining $A$ and $\lambda$ from our fit, we can predict $k_{\text {TET }}$ for any energy offset. We can then use our model to predict the PM behavior of other singlet-fission-ligand combinations in the search for better materials.

Designing PM Materials. The most useful PM material combination would couple to existing Si-PVs. Therefore, an ideal PM material must emit photons above the band gap of silicon and ideally with photon energies around $1.2 \mathrm{eV} \cdot{ }^{30,32}$ In Figure 6, we use our model to predict the PM behavior for QD-ligand systems with different ligand triplet energies. All the rate constants described above are kept constant, and only $k_{\text {TET }}$ is varied as a function of QD-ligand energy offset according to eq 3 . To predict the behavior of an optimized system, the intrinsic QD PLQEs is assumed to be $100 \%$, regardless of the band gap. The effect of surface trapping is also neglected, by setting $k_{\mathrm{q}}$ to zero. The only quenching of QD PLQE is therefore due to triplet energy transfer to the ligand. $k_{-\mathrm{TET}}$, the rate of triplet transfer to the ligand, is kept at $2.0 \times$ $10^{7} \mathrm{~s}^{-1}$, similar to the determined $k_{- \text {TET }}$ values. Further, the singlet-fission material is modeled to generate triplets with $185 \%$ efficiency, which is reasonable for an efficient singletfission material. ${ }^{55-57}$ To achieve a singlet-fission yield of $185 \%$, the rate of $\mathrm{SF}, k_{\mathrm{SF}}$, is increased to $8.6 \times 10^{8} \mathrm{~s}^{-1}$. The bimolecular rate constants $k_{\text {STET }}$ and $k_{- \text {STET }}$ are kept constant throughout the series. Therefore, the effect on PM behavior solely originates from the effect of ligand-QD interactions. 


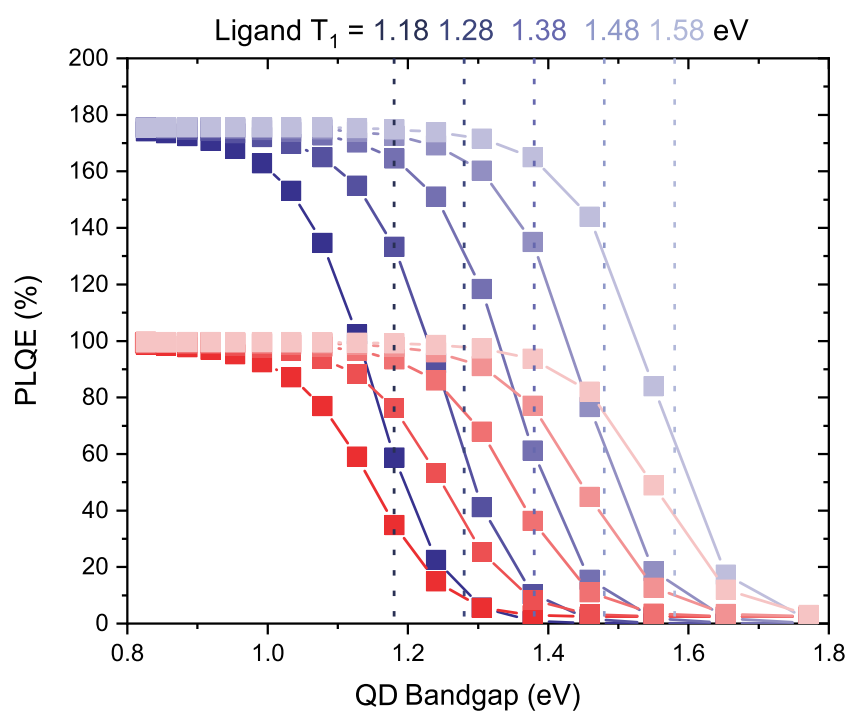

Figure 6. Modeling of an optimized photon multiplication (PM) material based on an absorbing singlet-fission material with $185 \%$ triplet yield. Triplet transfer to a PbS quantum dot is governed solely by the ligand triplet energy relative to the $\mathrm{QD}$ band gap. The energy difference between ligand and singlet-fission material is 0.1 $\mathrm{eV}$ as in the case for TIPS-Tc and TET-CA. Red squares correspond to direct excitation of the quantum dot, and the blue squares are the PM photoluminescence quantum efficiency when exciting the singlet-fission material.

Figure 6 shows the predicted PLQEs for systems with ligand $T_{1}$ energies ranging from 1.18 to $1.58 \mathrm{eV}$, for direct $\mathrm{QD}$ excitation (red) and SF excitation (blue). From the data in Figure 6 we can conclude that, to achieve a PM above $150 \%$ for a $1.2 \mathrm{eV} \mathrm{PbS} \mathrm{QD,} \mathrm{a} \mathrm{ligand} T_{1}$ energy of above $1.3 \mathrm{eV}$ is necessary. Assuming that the SF material also needs another $0.1 \mathrm{eV}$ to efficiently funnel triplets to the ligand, we propose that future SF materials with $T_{1}$ energies of $1.4-1.5 \mathrm{eV}$ will be required.

\section{CONCLUSIONS}

Here, we have answered a number of fundamental questions related to the triplet energy transfer from organic molecules to QDs. We have studied the energetic dependence of triplet energy transfer to $\mathrm{PbS}$ quantum dots. A photon multiplication scheme is used to estimate the efficiency of triplet transfer from steady-state PLQE measurements. Combined with the kinetic insight gained from ns-transient absorption measurements, we are able to extract the rate constants, $k_{\mathrm{TET}}$, of triplet energy transfer from a surface-bound TET-CA ligand to the PbS QD core. $k_{\mathrm{TET}}$ is found to follow an electron exchange mechanism, as described by Marcus-Hush theory, addressing the issue of how the triplet transfer is mediated. Interestingly, the triplet energy transfer in the reverse direction, from QD to ligand, occurs at a slower time scale than the QD ground state bleach recovery possibly indicating a stepwise process. ${ }^{26,27}$ We are also able to accurately model a singlet-fission-based photon multiplication process and conclude that an energetic driving force of $0.1 \mathrm{eV}$ is necessary for efficient net transfer to the QD. Furthermore, we highlight the need for singlet-fission materials with triplet energies around $1.4-1.5 \mathrm{eV}$ to achieve a photonmultiplication material matched to Si-PVs. Overall, we report a detailed thermodynamic and mechanistic study of the triplet energy transfer to $\mathrm{PbS} \mathrm{QDs}$, with implications for the future design of QD optoelectronic devices and photon multiplication devices.

\section{METHODS AND EXPERIMENTAL SECTION}

Chemicals. 5,12-Bis ((triisopropylsilyl)ethynyltetracene (TIPS-Tc; Figure S1, left) was purchased from Ark Pharm. 6,11-Bis((triisopropylsilyl)ethynyl)tetracene-2-carboxylic acid (TET-CA; Figure S1, right) was synthesized as described previously. ${ }^{25}$ All other material was bought from Sigma-Aldrich and used as received.

Quantum Dot Synthesis and Ligand Exchange. Synthesis of $\mathrm{PbS}$ QDs was carried out following the procedure by Hines and Scholes with modifications. ${ }^{41,58}$ In summary, $\mathrm{PbO}(0.45 \mathrm{~g})$, oleic acid (1.6-14 g, depending on the targeted band gap), and 1-octadecene $(10 \mathrm{~g})$ were degassed in a three necked flask at $110{ }^{\circ} \mathrm{C}$ for $2 \mathrm{~h}$. The temperature was then reduced to $95^{\circ} \mathrm{C}$. Under nitrogen, a solution of bis(trimethylsilyl)sulfide $(210 \mu \mathrm{L})$ in 1-octadecene $(5 \mathrm{~mL})$ was rapidly injected into the lead precursor solution. After cooling naturally to room temperature the $\mathrm{PbS}$ QDs were washed 4 times by precipitation/redispersion with acetone and hexane. The purified QDs were stored in a nitrogen-filled glovebox at high concentration $(>40 \mathrm{mg} / \mathrm{mL} />100 \mu \mathrm{M})$ until use.

Ligand exchange was carried out under nitrogen. QDs in toluene were diluted to $42 \mu \mathrm{M}$ in a toluene/THF mixture of $4: 1$. The ligand in $100 \mathrm{mg} / \mathrm{mL}$ THF solutions was added to the QD solution, keeping a ligand to QD mass ratio of 1:2 for all sizes of QDs. Ligand coverage and size distribution are summarized in the Supporting Information, Table S1.

Optical Spectroscopy. Steady-State Absorption. A Shimadzu UV3600Plus spectrometer was used to measure the absorbance spectra of the solutions. The high concentration of the PM solutions required samples to be measured in rectangular capillary tubes with a $200 \mu \mathrm{m}$ path length.

PLQE. The integrating sphere and PLQE measurement procedure has been described previously. ${ }^{15,59}$ An integrating sphere with a Spectralon-coated interior (Newport 819C-SL-5.3) was used for the absolute measurement. 515 and $658 \mathrm{~nm}$ laser diodes (Thorlabs) with a beam diameter at the sample of $3 \mathrm{~mm}$ were used as the excitation source. Light from the sphere was coupled into an Andor Kymera $328 \mathrm{i}$ spectrograph equipped with an InGaAs detector (Andor, iDus InGaAs 490). A NIST certified calibration lamp from Newport, 200 $\mathrm{W}$ quartz tungsten halogen lamp driven by an OPS-Q250 power supply, was used to generate a photons/count calibration file. The calibration file was generated by producing an irised beam of light from the calibration source into the integrating sphere; the spectral response was recorded with and without a series of long pass filters in order to resolve the longer wavelength response absent of the second harmonics from the shorter wavelengths. These were then also corrected for by measuring the transmission spectra of the filters. The calibration file was generated by comparing this data set to the calibrated spectrum of the lamp. To validate the calibration, a Rhodamine 6G sample in ethanol was measured in the sphere with $520 \mathrm{~nm}$ excitation. Three spectra were taken, with laser excitation on and off the sample and a blank sample. Each recorded spectra were multiplied with the generated correction curve to obtain the corrected spectra. From the corrected spectra, the PLQE was calculated to 92$95 \%$, in good agreement with the literature. ${ }^{60}$

ps-Transient Absorption. The short-time (fs-ns) transient absorption setup has been described previously. ${ }^{15}$ In summary, a light conversion PHAROS laser system with $400 \mu \mathrm{J}$ per pulse at 1030 $\mathrm{nm}$ with a repetition rate of $38 \mathrm{kHz}$ was used. The output is divided; one part is focused onto a $4 \mathrm{~mm}$ YAG substrate to produce the continuum probe beam from 520 to $950 \mathrm{~nm}$. The second part of the PHAROS output is led into a narrow band optical parametric oscillator system (ORPHEUS-LYRA, light conversion) outputting the pump beam. The probe pulse is delayed up to 2 ns with a mechanical delay-stage (Newport). A mechanical chopper (Thorlabs) is used to create an on-off pump-probe pulse series. The pump size on the sample is approximately $0.065 \mathrm{~mm}^{2}$ and the probe about $0.015 \mathrm{~mm}^{2}$. A silicon line scan camera (JAI SW-2000M-CL-80) fitted onto a 
visible spectrograph (Andor Solis, Shamrock) is used to record the transmitted probe light.

ns-Transient Absorption. The long-time ns-transient absorption setup has also been described previously. ${ }^{15}$ In short, the pump-probe setup consists of a probe from a LEUKOS Disco 1 UV super continuum laser (STM-1-UV, $1 \mathrm{kHz}$ ) and a pump generated in a TOPAS optical amplifier, pumped with the output from a SpectraPhysics Solstice Ace Ti:sapphire amplifier $(1 \mathrm{kHz})$. The probe beam is split into a reference and probe, and both are focused onto the sample. A pair of line image sensors (Hamamatsu, G11608) mounted on a spectrograph (Andor Solis, Shamrock SR303i) are used to detect the signal, using a custom built board from Stresing Entwickslungsburo to read out the signal.

\section{ASSOCIATED CONTENT}

\section{SI Supporting Information}

The Supporting Information is available free of charge at https://pubs.acs.org/doi/10.1021/acsnano.9b09339.

Additional details and data for the calculation of QD ligand coverage and size distribution. Additional data from the transient absorption measurements and details on data analysis and kinetic modeling (PDF)

Data underlying the figures and conclusions in this article is available at the University of Cambridge data repository at https://doi.org/10.17863/CAM50420.

\section{AUTHOR INFORMATION}

\section{Corresponding Author}

Akshay Rao - Cavendish Laboratory, University of Cambridge,

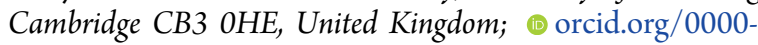
0003-4261-0766; Email: ar525@cam.ac.uk

\section{Authors}

Victor Gray - Cavendish Laboratory, University of Cambridge, Cambridge CB3 OHE, United Kingdom; Department of Chemistry—Ångström Laboratory, Uppsala University, 75120 Uppsala, Sweden; (i) orcid.org/0000-0001-6583-8654

Jesse R. Allardice - Cavendish Laboratory, University of Cambridge, Cambridge CB3 OHE, United Kingdom; (ㄱ) orcid.org/0000-0002-1969-7536

Zhilong Zhang - Cavendish Laboratory, University of Cambridge, Cambridge CB3 OHE, United Kingdom; (1) orcid.org/0000-0001-9903-4945

Simon Dowland - Cavendish Laboratory, University of Cambridge, Cambridge CB3 OHE, United Kingdom

James Xiao - Cavendish Laboratory, University of Cambridge, Cambridge CB3 OHE, United Kingdom

Anthony J. Petty, II - Department of Chemistry, University of Kentucky, Lexington, Kentucky 40506-0174, United States

John E. Anthony - Department of Chemistry, University of Kentucky, Lexington, Kentucky 40506-0174, United States; () orcid.org/0000-0002-8972-1888

Neil C. Greenham - Cavendish Laboratory, University of Cambridge, Cambridge CB3 OHE, United Kingdom; (1) orcid.org/0000-0002-2155-2432

Complete contact information is available at:

https://pubs.acs.org/10.1021/acsnano.9b09339

\section{Notes}

The authors declare no competing financial interest.

\section{ACKNOWLEDGMENTS}

We thank the Winton Programme for the Physics of Sustainability and the Engineering and Physical Sciences Research Council for funding. This project has received funding from the European Research Council (ERC) under the European Union's Horizon 2020 research and innovation programme (grant agreement 758826). V.G. acknowledges funding from the Swedish research council, Vetenskapsrådet 2018-00238. J.R.A. acknowledges Cambridge Commonwealth European and International Trust for financial support. Z.Z. acknowledges funding from the European Union's Horizon 2020 research and innovation programme under the Marie Skłodowska-Curie Actions grant (842271-TRITON project). J.X. acknowledges EPSRC Cambridge NanoDTC, EP/ L015978/1 for financial support. J.E.A. and A.P. acknowledge the U.S. National Science Foundation (DMREF-1627428) for support of organic semiconductor synthesis.

\section{REFERENCES}

(1) Kamat, P. V. Quantum Dot Solar Cells. Semiconductor Nanocrystals as Light Harvesters. J. Phys. Chem. C 2008, 112, 18737-18753.

(2) Kamat, P. V. Quantum Dot Solar Cells. The Next Big Thing in Photovoltaics. J. Phys. Chem. Lett. 2013, 4, 908-918.

(3) Dai, X.; Zhang, Z.; Jin, Y.; Niu, Y.; Cao, H.; Liang, X.; Chen, L.; Wang, J.; Peng, X. Solution-Processed, High-Performance LightEmitting Diodes Based on Quantum Dots. Nature 2014, 515, 96-99.

(4) Sargent, E. H. Colloidal Quantum Dot Solar Cells. Nat. Photonics 2012, 6, 133-135.

(5) Zhang, Z.; Yang, J.; Wen, X.; Yuan, L.; Shrestha, S.; Stride, J. A.; Conibeer, G. J.; Patterson, R. J.; Huang, S. Effect of Halide Treatments on PbSe Quantum Dot Thin Films: Stability, Hot Carrier Lifetime, and Application to Photovoltaics. J. Phys. Chem. C 2015, 119, 24149-24155.

(6) Zhang, Z.; Chen, Z.; Zhang, J.; Chen, W.; Yang, J.; Wen, X.; Wang, B.; Kobamoto, N.; Yuan, L.; Stride, J. A.; Conibeer, G. J.; Patterson, R. J.; Huang, S. Significant Improvement in the Performance of PbSe Quantum Dot Solar Cell by Introducing a $\mathrm{CsPbBr} 3$ Perovskite Colloidal Nanocrystal Back Layer. Adv. Energy Mater. 2017, 7, 1601773.

(7) Zhang, Z.; Chen, Z.; Yuan, L.; Chen, W.; Yang, J.; Wang, B.; Wen, X.; Zhang, J.; Hu, L.; Stride, J. A.; Conibeer, G. J.; Patterson, R. J.; Huang, S. A New Passivation Route Leading to Over 8\% Efficient PbSe Quantum-Dot Solar Cells via Direct Ion Exchange with Perovskite Nanocrystals. Adv. Mater. 2017, 29, 1703214.

(8) Pan, Z.; Rao, H.; Mora-Seró, I.; Bisquert, J.; Zhong, X. Quantum Dot-Sensitized Solar Cells. Chem. Soc. Rev. 2018, 47, 7659-7702.

(9) Efros, A. L.; Rosen, M.; Kuno, M.; Nirmal, M.; Norris, D.; Bawendi, M. Band-Edge Exciton in Quantum Dots of Semiconductors with a Degenerate Valence Band: Dark and Bright Exciton States. Phys. Rev. B: Condens. Matter Mater. Phys. 1996, 54, 4843-4856.

(10) Scholes, G. D.; Kim, J.; Wong, C. Y.; Huxter, V. M.; Nair, P. S.; Fritz, K. P.; Kumar, S. Nanocrystal Shape and the Mechanisme of Exciton Spin Relaxation. Nano Lett. 2006, 6, 1765-1771.

(11) Tabachnyk, M.; Ehrler, B.; Gélinas, S.; Böhm, M. L.; Walker, B. J.; Musselman, K. P.; Greenham, N. C.; Friend, R. H.; Rao, A. Resonant Energy Transfer of Triplet Excitons from Pentacene to PbSe Nanocrystals. Nat. Mater. 2014, 13, 1033-1038.

(12) Thompson, N. J.; Wilson, M. W. B.; Congreve, D. N.; Brown, P. R.; Scherer, J. M.; Bischof, T. S.; Wu, M.; Geva, N.; Welborn, M.; Voorhis, T. V.; Bulovic, V.; Bawendi, M. G.; Baldo, M. A. Energy Harvesting of Non-Emissive Triplet Excitons in Tetracene by Emissive PbS Nanocrystals. Nat. Mater. 2014, 13, 1039-1043.

(13) Mongin, C.; Moroz, P.; Zamkov, M.; Castellano, F. N. Thermally Activated Delayed Photoluminescence from PyrenylFunctionalized CdSe Quantum Dots. Nat. Chem. 2018, 10, 225-230. 
(14) Davis, N. J. L. K.; Allardice, J. R.; Xiao, J.; Petty, A. J.; Greenham, N. C.; Anthony, J. E.; Rao, A. Singlet Fission and Triplet Transfer to PbS Quantum Dots in TIPS-Tetracene Carboxylic Acid Ligands. J. Phys. Chem. Lett. 2018, 9, 1454-1460.

(15) Allardice, J. R.; Thampi, A.; Dowland, S.; Xiao, J.; Gray, V.; Zhang, Z.; Budden, P.; Petty, A. J.; Davis, N. J. L. K.; Greenham, N. C.; Anthony, J. E.; Rao, A. Engineering Molecular Ligand Shells on Quantum Dots for Quantitative Harvesting of Triplet Excitons Generated by Singlet Fission. J. Am. Chem. Soc. 2019, 141, 1290712915 .

(16) Mongin, C.; Garakyaraghi, S.; Razgoniaeva, N.; Zamkov, M.; Castellano, F. N. Direct Observation of Triplet Energy Transfer from Semiconductor Nanocrystals. Science 2016, 351, 369-372.

(17) Huang, Z.; Li, X.; Mahboub, M.; Hanson, K. M.; Nichols, V. M.; Le, H.; Tang, M. L.; Bardeen, C. J. Hybrid Molecule-Nanocrystal Photon Upconversion across the Visible and Near-Infrared. Nano Lett. 2015, 15, 5552-5557.

(18) Jiang, Y.; Wang, C.; Rogers, C. R.; Kodaimati, M. S.; Weiss, E. A. Regio- and Diastereoselective Intermolecular $[2+2]$ Cycloadditions Photocatalysed by Quantum Dots. Nat. Chem. 2019, 11, 1034-1040.

(19) Huang, Z.; Tang, M. L. Designing Transmitter Ligands That Mediate Energy Transfer between Semiconductor Nanocrystals and Molecules. J. Am. Chem. Soc. 2017, 139, 9412-9418.

(20) Piland, G. B.; Huang, Z.; Lee Tang, M.; Bardeen, C. J. Dynamics of Energy Transfer from CdSe Nanocrystals to Triplet States of Anthracene Ligand Molecules. J. Phys. Chem. C 2016, 120, 5883-5889.

(21) Li, X.; Huang, Z.; Zavala, R.; Tang, M. L. Distance-Dependent Triplet Energy Transfer between CdSe Nanocrystals and Surface Bound Anthracene. J. Phys. Chem. Lett. 2016, 7, 1955-1959.

(22) Wang, Y. A.; Li, J. J.; Chen, H.; Peng, X. Stabilization of Inorganic Nanocrystals by Organic Dendrons. J. Am. Chem. Soc. 2002, 124, 2293-2298.

(23) Mahboub, M.; Huang, Z.; Tang, M. L. Efficient Infrared-toVisible Upconversion with Subsolar Irradiance. Nano Lett. 2016, 16, 7169-7175.

(24) Huang, Z.; Xu, Z.; Mahboub, M.; Li, X.; Taylor, J. W.; Harman, W. H.; Lian, T.; Tang, M. L. PbS/CdS Core-Shell Quantum Dots Suppress Charge Transfer and Enhance Triplet Transfer. Angew. Chem., Int. Ed. 2017, 56, 16583-16587.

(25) Kroupa, D. M.; Arias, D. H.; Blackburn, J. L.; Carroll, G. M.; Granger, D. B.; Anthony, J. E.; Beard, M. C.; Johnson, J. C. Control of Energy Flow Dynamics between Tetracene Ligands and $\mathrm{PbS}$ Quantum Dots by Size Tuning and Ligand Coverage. Nano Lett. 2018, 18, 865-873.

(26) Garakyaraghi, S.; Mongin, C.; Granger, D. B.; Anthony, J. E.; Castellano, F. N. Delayed Molecular Triplet Generation from Energized Lead Sulfide Quantum Dots. J. Phys. Chem. Lett. 2017, 8, $1458-1463$

(27) Bender, J. A.; Raulerson, E. K.; Li, X.; Goldzak, T.; Xia, P.; Van Voorhis, T.; Tang, M. L.; Roberts, S. T. Surface States Mediate Triplet Energy Transfer in Nanocrystal-Acene Composite Systems. J. Am. Chem. Soc. 2018, 140, 7543-7553.

(28) Luo, X.; Han, Y.; Chen, Z.; Li, Y.; Liang, G.; Liu, X.; Ding, T.; Nie, C.; Wang, M.; Castellano, F. N.; Wu, K. Mechanisms of Triplet Energy Transfer across the Inorganic Nanocrystal/Organic Molecule Interface. Nat. Commun. 2020, 11, 28.

(29) Smith, M. B.; Michl, J. Singlet Fission. Chem. Rev. 2010, 110, 6891-6936

(30) Rao, A.; Friend, R. H. Harnessing Singlet Exciton Fission to Break the Shockley-Queisser Limit. Nat. Rev. Mater. 2017, 2, 17063.

(31) Lee, J.; Jadhav, P.; Reusswig, P. D.; Yost, S. R.; Thompson, N. J.; Congreve, D. N.; Hontz, E.; Van Voorhis, T.; Baldo, M. A. Singlet Exciton Fission Photovoltaics. Acc. Chem. Res. 2013, 46, 1300-1311.

(32) Futscher, M. H.; Rao, A.; Ehrler, B. The Potential of Singlet Fission Photon Multipliers as an Alternative to Silicon-Based Tandem Solar Cells. ACS Energy Lett. 2018, 3, 2587-2592.
(33) Ehrler, B.; Musselman, K. P.; Böhm, M. L.; Friend, R. H.; Greenham, N. C. Hybrid Pentacene/a-Silicon Solar Cells Utilizing Multiple Carrier Generation via Singlet Exciton Fission. Appl. Phys. Lett. 2012, 101, 2010-2013.

(34) Ehrler, B.; Wilson, M. W. B.; Rao, A.; Friend, R. H.; Greenham, N. C. Singlet Exciton Fission-Sensitized Infrared Quantum Dot Solar Cells. Nano Lett. 2012, 12, 1053-1057.

(35) Reusswig, P. D.; Congreve, D. N.; Thompson, N. J.; Baldo, M. A. Enhanced External Quantum Efficiency in an Organic Photovoltaic Cell via Singlet Fission Exciton Sensitizer. Appl. Phys. Lett. 2012, 101, 113304.

(36) Wu, T. C.; Thompson, N. J.; Congreve, D. N.; Hontz, E.; Yost, S. R.; Van Voorhis, T.; Baldo, M. A. Singlet Fission Efficiency in Tetracene-Based Organic Solar Cells. Appl. Phys. Lett. 2014, 104, 193901.

(37) Thompson, N. J.; Hontz, E.; Congreve, D. N.; Bahlke, M. E.; Reineke, S.; Van Voorhis, T.; Baldo, M. A. Nanostructured Singlet Fission Photovoltaics Subject to Triplet-Charge Annihilation. Adv. Mater. 2014, 26, 1366-1371.

(38) Dexter, D. L. Two Ideas on Energy Transfer Phenomena: IonPair Effects Involving the $\mathrm{OH}$ Stretching Mode, and Sensitization of Photovoltaic Cells. J. Lumin. 1979, 18-19, 779-784.

(39) MacQueen, R.; Liebhaber, M.; Niederhausen, J.; Mews, M.; Gersmann, C.; Jäckle, S.; Jäger, K.; Tayebjee, M.; Jehangir, Y.; Schmidt, T. W.; Rech, B.; Lips, K. Crystalline Silicon Solar Cells with Tetracene Interlayers: The Path to Silicon-Singlet Fission Heterojunction Devices. Mater. Horiz. 2018, 5, 1065-1075.

(40) Einzinger, M.; Wu, T.; Kompalla, J. F.; Smith, H. L.; Perkinson, C. F.; Nienhaus, L.; Wieghold, S.; Congreve, D. N.; Kahn, A.; Bawendi, M. G.; Baldo, M. A. Sensitization of Silicon by Singlet Exciton Fission in Tetracene. Nature 2019, 571, 90-94.

(41) Hines, M. A.; Scholes, G. D. Colloidal PbS Nanocrystals with Size-Tunable Near-Infrared Emission: Observation of Post-Synthesis Self-Narrowing of the Particle Size Distribution. Adv. Mater. 2003, 15, 1844-1849.

(42) Semonin, O. E.; Johnson, J. C.; Luther, J. M.; Midgett, A. G.; Nozik, A. J.; Beard, M. C. Absolute Photoluminescence Quantum Yields of IR-26 Dye, PbS, and PbSe Quantum Dots. J. Phys. Chem. Lett. 2010, 1, 2445-2450.

(43) Stern, H. L.; Musser, A. J.; Gelinas, S.; Parkinson, P.; Herz, L. M.; Bruzek, M. J.; Anthony, J.; Friend, R. H.; Walker, B. J. Identification of a Triplet Pair Intermediate in Singlet Exciton Fission in Solution. Proc. Natl. Acad. Sci. U. S. A. 2015, 112, 7656-7661.

(44) Stern, H. L.; Cheminal, A.; Yost, S. R.; Broch, K.; Bayliss, S. L.; Chen, K.; Tabachnyk, M.; Thorley, K.; Greenham, N.; Hodgkiss, J. M.; Anthony, J.; Head-Gordon, M.; Musser, A. J.; Rao, A.; Friend, R H. Vibronically Coherent Ultrafast Triplet-Pair Formation and Subsequent Thermally Activated Dissociation Control Efficient Endothermic Singlet Fission. Nat. Chem. 2017, 9, 1205-1212.

(45) Gray, V.; Dreos, A.; Erhart, P.; Albinsson, B.; Moth-Poulsen, K.; Abrahamsson, M. Loss Channels in Triplet-Triplet Annihilation Photon Upconversion: Importance of Annihilator Singlet and Triplet Surface Shapes. Phys. Chem. Chem. Phys. 2017, 19, 10931-10939.

(46) Albinsson, B.; Mårtensson, J. Long-Range Electron and Excitation Energy Transfer in Donor-Bridge-Acceptor Systems. J. Photochem. Photobiol., C 2008, 9, 138-155.

(47) Subotnik, J. E.; Vura-weis, J.; Sodt, A. J.; Ratner, M. A. Predicting Accurate Electronic Excitation Transfer Rates via Marcus Theory with Boys or Edmiston-Ruedenberg Localized Diabatization. J. Phys. Chem. A 2010, 114, 8665-8675.

(48) Closs, G. L.; Piotrowiak, P.; MacInnis, J. M.; Fleming, G. R. Determination of Long Distance Intramolecular Triplet Energy Transfer Rates. A Quantitative Comparison with Electron Transfer. J. Am. Chem. Soc. 1988, 110, 2652-2653.

(49) Closs, G. L.; Piotrowiak, P.; Johnson, M. D.; Miller, J. R. A Connection between Intramolecular Long-Range Electron, Hole, and Triplet Energy Transfers. J. Am. Chem. Soc. 1989, 111, 3751-3753.

(50) Tisdale, W. A.; Zhu, X. Y. Artificial Atoms on Semiconductor Surfaces. Proc. Natl. Acad. Sci. U. S. A. 2011, 108, 965-970. 
(51) Marcus, R. A. Chemical and Electrochemical Electron-Transfer Theory. Annu. Rev. Phys. Chem. 1964, 15, 155-196.

(52) Balzani, V.; Bolletta, F.; Scandola, F. Vertical and Nonvertical" Energy Transfer Processes. A General Classical Treatment ". J. Am. Chem. Soc. 1980, 102, 2152-2163.

(53) Sudha Devi, L.; Al-Suti, M. K.; Dosche, C.; Khan, M. S.; Friend, R. H.; Köhler, A. Triplet Energy Transfer in Conjugated Polymers. I. Experimental Investigation of a Weakly Disordered Compound. Phys. Rev. B: Condens. Matter Mater. Phys. 2008, 78, 045210.

(54) Hoffmann, S. T.; Scheler, E.; Koenen, J. M.; Forster, M.; Scherf, U.; Strohriegl, P.; Bässler, H.; Köhler, A. Triplet Energy Transfer in Conjugated Polymers. III. An Experimental Assessment Regarding the Influence of Disorder on Polaronic Transport. Phys. Rev. B: Condens. Matter Mater. Phys. 2010, 81, 165208.

(55) Johnson, J. C.; Nozik, A. J.; Michl, J. High Triplet Yield from Singlet Fission in a Thin Film of 1,3-Diphenylisobenzofuran. J. Am. Chem. Soc. 2010, 132, 16302-16303.

(56) Walker, B. J.; Musser, A. J.; Beljonne, D.; Friend, R. H. Singlet Exciton Fission in Solution. Nat. Chem. 2013, 5, 1019-1024.

(57) Sanders, S. N.; Kumarasamy, E.; Pun, A. B.; Trinh, M. T.; Choi, B.; Xia, J.; Taffet, E. J.; Low, J. Z.; Miller, J. R.; Roy, X.; Zhu, X. Y.; Steigerwald, M. L.; Sfeir, M. Y.; Campos, L. M. Quantitative Intramolecular Singlet Fission in Bipentacenes. J. Am. Chem. Soc. 2015, 137, 8965-8972.

(58) Zhang, J.; Crisp, R. W.; Gao, J.; Kroupa, D. M.; Beard, M. C.; Luther, J. M. Synthetic Conditions for High-Accuracy Size Control of PbS Quantum Dots. J. Phys. Chem. Lett. 2015, 6, 1830-1833.

(59) De Mello, J. C.; Wittmann, H. F.; Friend, R. H. An Improved Experimental Determination of External Photoluminescence Quantum Efficiency. Adv. Mater. 1997, 9, 230-232.

(60) Kubin, R. F.; Fletcher, A. N. Fluorescence Quantum Yields of Some Rhodamine Dyes. J. Lumin. 1982, 27, 455-462. 